\title{
Inmediatez y fact-checking: análisis del portal Ecuador Chequea
}

\section{Immediacy and fact-checking: analysis from the Ecuador Chequea portal ${ }^{1}$}

\section{Gabriela Lourdes Vélez Bermello}

Facultad de Ciencias Sociales y de la Salud, Universidad Estatal Península de Santa Elena, Ecuador

gvelezb@upse.edu.ec

Recibido: 07/11/2019 • Aceptado: 15/11/2020

\section{RESUMEN}

Las noticias falsas y la desinformación han sido, desde tiempos memorables, temas de debates en los medios de comunicación. Estos se ven enfrascados por la inmediatez en el proceso de la construcción de la realidad y los intereses, por señalar algunos de los condicionantes que el sistema genera. Internet actúa como un "arma de doble filo"; por un lado, se evidencia una gran avalancha de información y, por otro, la carencia de credibilidad en los portales noticiosos. Por este motivo, la práctica del fact-checking se va posicionando aunque con divergentes metodologías y técnicas. Esta investigación propone un estado de los estudios generados con relación al fact-checking, para luego ofrecer un análisis de la periodicidad de contenidos que se publican en el portal web Ecuador Chequea, indagando en las distintas fuentes a las que recurre el equipo de chequeo, con el objetivo de clasificar si la información tiene o no veracidad. Con el respaldo de bases teóricas y aplicando la observación sistematizada, este trabajo

1 Este artículo es parte de las primeras investigaciones de tesis doctoral de la autora como estudiante de la Universidad Nacional de Rosario y cursante del doctorado en Comunicación Social. 
aporta a las reflexiones en torno al tratamiento de noticias en el primer portal de verificación de información de Ecuador.

Palabras clave: Verificación de hechos, fake news, comunicación, construcción de la realidad, periodismo de verificación.

\section{ABSTRACT}

Fake news and disinformation have long been the subject of debate in the media. These are engulfed by the immediate in the process of construction of reality and interests, to point out some of the conditions that the system generates. The Internet acts as a "double-edged sword"; on the one hand, a great avalanche of information is evident and, on the other, the lack of credibility in the news portals. For this reason, the practice of data verification is positioning itself, although with divergent methodologies and techniques. This research proposes a status of the studies generated in relation to the fact-check, to then offer an analysis of the periodicity of contents that are published on the Ecuador Chequea web portal, inquiring into the different sources used by the checking team, in order to classify whether or not the information is true. With the support of theoretical bases and applying systematic observation, this work contributes to the reflections on the treatment of news in the first information verification portal in Ecuador.

Keywords: Fact verification, Fake news, Communication, Construction of reality, Verification journalism.

\section{INTRODUCCIÓN}

El periodismo de verificación de datos, también conocido por su palabra en inglés como fact-checking, nació en el 2003 en Estados Unidos con la creación del portal FactCheck.org. En ese entonces, el periodista Brooks Jackson se instaló en la Universidad de Pensilvania, luego de años de trabajar en CNN (Zommer, 2015).

Elisabeth (2014) define al fact-checking como una actividad que realizan varias organizaciones y que se dedican exclusivamente al periodismo de verificación de contenidos de los discursos políticos o del chequeo de las publicaciones que hacen los medios de comunicación. Dichas 
entidades de chequeo explican que su trabajo está libre de intereses concretos y de retóricas.

El fact-checking ha ganado espacio en los últimos años, sin embargo, dentro del ejercicio profesional periodístico ya se conocía la actividad de chequeo. Esto porque en 1990 surgió la denominación de periodismo de verificación, que se practicaba y se practica como parte de las rutinas productivas y de los filtros editoriales que se desarrollan en los medios de comunicación convencionales (Rodríguez-Pérez, 2020). No obstante, el periodismo de verificación poco a poco fue desligándose para consolidarse como un ejercicio que se emplea en medios específicos y se ha ido propagando en todo el mundo, especialmente en Latinoamérica, donde la mayoría de las instituciones de fact-checking nacen desenlazados de los medios convencionales y se presentan actualmente como un nuevo ecosistema o una rama alterna al periodismo (Palau-Sampio, 2018).

Ufarte-Ruiz et al. (2018), consideran que las funciones de fact-checking son necesarias frente al periodismo que ha dejado las labores de reportar en las calles para centrarse en un trabajo más de escritorio. Además, aseguran que con la aparición del fact-checking existen más ofertas de trabajo y, con ello, la adaptación de nuevas habilidades y competencias para poder ejercerlo. Esto último también es considerado por otros autores como Graves (2018), quien mira al periodismo de verificación como la recuperación del ofrecimiento de contenidos apegados a la veracidad y que esté contrastado de la mano de un personal de trabajo idóneo para este ámbito.

Según un listado de la Universidad de Duke, las iniciativas de medios de verificación han aumentado en todo el mundo a 188 en más de 60 países (Stencel, 2019). Por su parte, la International Fact-Checking Network (IFCN) tiene registrados a 90 signatarios verificados con el código de principios ${ }^{2}$ IFCN (IFCN, 2020). Además, en el marco de la pandemia COVID-19, la Fundación Gabo destacó el 2 de abril, Día Internacional de la Verificación de Hechos, que existen más de 100 sitios dedicados a la verificación de información en torno al virus (Fundación Gabo, 2020).

2 Los principios del IFCN pueden consultarse en: https://ifcncodeofprinciples.poynter.org/know-more/ the-commitments-of-the-code-of-principles 
La acción de verificar y contrastar información diferencia el periodismo convencional del que se realiza en el fact-checking. En el primero, se procede a evaluar los contenidos antes de ser publicados; mientras que, en el segundo, se hace después de que se haya difundido una información. Por lo tanto, el fact-checking activa un escrutinio de contenidos de dudosa procedencia y que hayan generado controversia en diversos grupos sociales.

Esta característica diferenciadora ha causado serios cuestionamientos de las prácticas del periodismo convencional, aludiendo que la inmediatez de la información ha relegado a la verificación de los hechos a un segundo plano en las redacciones. De este modo, Luna (2015) devela la existencia de un desafío direccionado a los medios de comunicación en su reto de brindar mejores contenidos antes que la inmediatez: "Hay que ser minuciosos por parte del periodista editor, en contrastar la información y tener el rigor de verificar cada información, ya que la inmediatez puede cobrar el prestigio del medio o del periodista" (Luna, 2015, p. 49).

En este mismo registro, Martini (2000) sostiene que la verificación de los hechos y el contraste de información son indispensables para cualquier medio comunicativo. Con ello, hace referencia a la necesidad que tienen los medios de concretar qué es noticia, cómo surge ese acontecimiento noticioso, bajo qué sistemas de clasificación pasa un hecho que desea ser publicado, cómo se ajustan los criterios de noticiabilidad y de qué manera se manejan los supuestos noticiosos que nacen desde la opinión pública. Estos son aspectos obviados en muchas redacciones dentro de sus rutinas productivas y que limitan los horizontes y/o alcances que debe tener el periodismo (Martini, 2000).

Lo explicado en el párrafo anterior se enlaza a lo que Wolf (1997) resalta en cuanto al peso de información para traducirse en una publicación. En otras palabras, el valor-noticia y, sin bien hace años Internet no había ganado el auge que tiene en la actualidad, el periodismo también padecía de filtraciones de información a medias, no contrastada e incluso falsa.

A medida que el periodismo convencional perdía fuerza por la falta de rigor en el proceso de verificación, el fact-checking se fue posicionando debido a que, con Internet, las noticias falsas, también llamadas fake news por su denominación en inglés, se propagaron con más intensidad. 
Dichas noticias falsas han sido categorizadas por García-Galera et al. (2020) de la siguiente forma: "1. Noticia inventada por la fuente, 2. Noticia falseada por la fuente y 3 . Noticia errada por falta de rigor profesional" (García-Galera et al. 2020, p. 113). Dentro de estos aspectos de desinformación, Salaverría et al. (2020) han destacado cuatro tipos de bulos que son: broma, exageración, descontextualización y engaño; en ese mismo orden se establecen desde los más leves hasta los más graves.

Por su parte, como parte del proceso de la desinformación se consideran los siguientes tres elementos: el tipo de contenido falso publicado, el modo de difusión y la motivación de los creadores. Estos elementos fueron establecidos por Claire Wardle (2017), creadora de First Draft y una de las impulsadoras de fact-checking.

Con la proliferación de Internet, el crecimiento de noticias falsas y la carencia de credibilidad han ido en aumento. Según el Informe sobre Medición de la Sociedad de la Información del 2018 de la Unión Internacional de Telecomunicaciones (ITU): "A finales de 2018, el 51,2\% de las personas, es decir, 3900 millones, utilizaban Internet" (ITU, 2018, p. 2). Con ello, es exponencial la cantidad de información que dichos usuarios generan y que puede causar saturación.

En el otro extremo están los usuarios, quienes ante la infoxicación ${ }^{3}$ que existe en la web, no logran discernir qué es creíble o no; incluso, no se dan tiempo para reconocer qué datos son reales al consumir información de manera vertiginosa. Zygmunt Bauman (2015) acredita esta forma vertiginosa de vivir a la modernidad líquida, donde todo se consume, desde las cosas materiales hasta las experiencias.

A modo de ejemplo, según el diario español La Vanguardia, mensualmente existen 2230 millones de usuarios en Facebook (Pons, 2018). Anexo a esto, la plataforma Techcrunch publica que en dicha red social se suben "2.5 mil millones de piezas de contenido y más de 500 terabytes ingeridos todos los días" (Constine, 2012). Dentro de este volumen de información hay fake news, bulos, mentiras y una avalancha de desinformación.

3 El término de infoxicación como tal fue acuñado por vez primera por Alfons Cornella. Se refiere al exceso informacional en la que hay más información para procesar de la que humanamente se puede. 
ITU afirma que "los usuarios de computadoras en los países desarrollados parecen poseer más conocimientos sobre las TIC que los usuarios de los países en desarrollo, lo que indica una grave limitación del potencial de desarrollo de los países en desarrollo y los PMA" (ITU, 2018, p. 3). Sin embargo, el hecho de conocer más sobre las TIC no implica discernir la información veraz.

Bajo este contexto de inmediatez, actúa el fact-checking, una actividad que practica una metodología que también ha sido discutida por varios investigadores.

Zommer (2015), considerada la precursora del fact-checking en Latinoamérica, indica que la verificación está conformada por 4 etapas y 8 pasos, las cuales se distribuyen así como se detalla en la Tabla 1.

Tabla 1

Etapas de fact-checking

\begin{tabular}{llll}
\hline ETAPA 1 & ETAPA 2 & ETAPA 3 & ETAPA 4 \\
\hline 1. Elegir una frase & $\begin{array}{l}\text { 3. Consultar a la } \\
\text { fuente original }\end{array}$ & $\begin{array}{l}\text { 6. Ubicar en } \\
\text { contexto }\end{array}$ & $\begin{array}{l}\text { 7. Confirmar, } \\
\text { relativizar o } \\
\text { desmentir la } \\
\text { afirmación }\end{array}$ \\
$\begin{array}{l}\text { 2. Ponderar su } \\
\text { relevancia }\end{array}$ & $\begin{array}{l}\text { 4. Consultar a la/s } \\
\text { fuente/s oficial/es } \\
\text { 5. Consultar a fuentes } \\
\text { alternativas }\end{array}$ & & \\
\hline
\end{tabular}

Nota. Elaboración propia basado en Zommer (2015).

La lógica que sigue este método consiste en que puede verificarse lo que salió en un medio de comunicación, pero también un discurso de algún político. Luego, los medios dedicados a chequeo comienzan a investigar y, finalmente, se efectúa la publicación con datos contrastados. Estos pasos y similares características manejan varios medios de chequeo, así lo confirman Vizoso \& Vázquez-Herrero (2019), quienes analizaron 19 proyectos de este tipo y obtuvieron como resultados semejantes estructuras en cuanto a las metodlogías, sobre todo en Sudamérica.

Zommer (2014) agrega que el primer paso dentro de la primera etapa es seleccionar una frase del ámbito público; este paso se efectúa tomando en 
cuenta que cuestiones relacionadas con la religión, farándula, deportes y otros, no son objeto de chequeo.

En el segundo paso de la primera etapa, se pondera su relevancia. "Aunque se utilizan tres criterios claros para definir las frases a analizar, se trata de una decisión editorial" (Zommer, 2014, p. 35). La elección de la frase por su relevancia pone en duda el proceso metodológico y su objetividad.

Es importante, en esta fase, aclarar que dicha objetividad se enmarca a lo que se denomina como "Ideología de la Objetividad", como si fuera un principio de imparcialidad, que aunque se cita como decadente en estos tiempos, aún sigue en vigencia en el proceso de rutinas productivas en los medios. Lo anterior resaltado por Becerra y Marino (2014) quienes refieren que es:

Un equilibrio editorial en la ponderación de diferentes fuentes y perspectivas. Esa "ideología de la objetividad" corresponde a una etapa extendida en distintos países, donde las empresas periodísticas promovían un ethos que premiaba el contraste entre varias fuentes informativas como un indicador de calidad. Ese paradigma está en decadencia pues el modelo de "periodismo partisano", faccioso y eximido de la obligación de verificar con varias fuentes lo que se afirma, resulta eficaz para lograr impactos más excitantes en las audiencias y más económico, ya que involucra menor cantidad de recursos para la resolución de notas (Becerra \& Marino, 2014, p. 3).

Las siguientes etapas y pasos del fact-checking están ligadas a las consultas de fuentes, a contextualizar y a calificar. Este sistema, que sería una especie de rutina productiva, se ve justificado por la cantidad de fake news que han existido y existen hasta la actualidad, mucho más potencializadas con Internet.

A partir de aquí, han surgido algunos estudios y cuestionamientos, puesto que esta metodología se asemeja en los otros medios de chequeo. Por ejemplo, Rodríguez-Pérez (2020) sostiene que hay cierta desconfianza en los procesos metodológicos del fact-checking. Esto da pauta a reordenar los principios generados en esta actividad. 
En este contexto, los medios de verificación se encuentran en la encrucijada de erigirse en un poderoso ecosistema que, junto con las alianzas que pueda tejer, sea capaz de luchar contra la desinformación; y, por otro lado, legitimarse socialmente y vencer la desconfianza ciudadana hacia los medios de comunicación (Rodríguez-Pérez, 2020, p. 254).

Para Uscinski y Butler (2013) existe una insostenibilidad de la epistemología en cuanto al funcionamiento de verificación de hechos y, el mismo Uscinski (2015), presenta después otro análisis donde asevera que existe inocencia en esta nueva rama del periodismo, llamada fact-checking.

La revista The New Yorker posee un equipo de fact checkers, quienes aplican una lógica diferente con el fin de garantizar la calidad de lo que se difunde con chequeos de los hechos realizados en los textos antes de su puesta en circulación. Esta práctica no está dilatada en Latinoamérica, donde pocos medios hacen lo mismo, como en el caso de la Revista Piaui de Brasil, que cuenta con una fact checker al interior de su redacción (Zommer, 2015)

En Europa, un estudio realizado por García-Galera et al. (2020), donde fueron analizados dos portales web de chequeo, se resalta que las funciones de fact-checking son semejantes a algunas actividades ejercidas en el periodismo tradicional. Por ello y otros aspectos se incita a impulsar nuevas iniciativas de verificación.

Buena parte de las detecciones de casos de desinformación hechas desde las plataformas de verificación y fact-checking Maldita.es y Newtral surgen ante las sospechas de los usuarios sobre aquello que les llega por redes sociales o servicios de mensajería instantánea como WhatsApp, de tal modo que tendrían un papel similar al de los lectores en la prensa tradicional desde la sección de Cartas al director. Uno de los casos explicado en este artículo respalda el paralelismo y la conveniencia de considerar en un futuro esa sección como base de la búsqueda de textos periodísticos cuyos errores hubieran sido puestos en evidencia por los lectores (García-Galera et al., 2020, p. 116). 
Otro punto a considerar en la investigación citada es que tanto los portales Newtral como Maldita.es no poseen un inventario sistematizado de fake news producidas por medios periodísticos profesionales; es decir que estos portales no clasifican las noticias falsas en función del medio que las publicó, por lo que su modo de proceder consiste en interactuar con los seguidores que van alimentando su acopio con mensajes dudosos vistos en las redes, por lo que el trabajo habitual de verificación viene explícito por la propia viralidad de los bulos y falsedades que inundan el espacio público digital. Posteriormente, comienzan el proceso de verificación, donde los pasos desarrollados hacia la consulta a las fuentes aún no garantizan la total credibilidad de los mencionados portales.

En una investigación efectuada en seis países de Latinoamérica se estudió un total de nueve proyectos de chequeo que iniciaron en el 2010. Este proceso realizado por Palau-Sampio (2018) reveló la existencia de desfases entre las verificaciones y la presentación de los resultados. La autora sugiere la necesidad de mejorar la cantidad de fuentes de contraste, así como incluir voces de expertos e interacción con los lectores.

La investigadora Busaniche (2005) hace una mirada a la difusa imagen de controlar las ideas en un mundo de abundante creación de tecnologías, por lo que habría que profundizar si el fact-checking es la solución a futuro en la búsqueda por la credibilidad de los hechos o es la muestra exacta del temor en la era de Internet. Para Busaniche (2005) existen nuevas tendencias de comunicación que van marcadas por la digitalización, con personas que generan sus propios contenidos y se incorporan a diversas redes. Todos estos fenómenos están creando fuertes resistencias en aquellos que ven peligrar sus negocios o en aquellos que sencillamente le tienen temor a la libertad y a la descentralización casi anárquica que propone la red.

No obstante, hay otros investigadores que resaltan las propuestas vertidas desde el fact-checking, como en el caso de Echevarría (2017), quien hace una profunda observación sobre la falta de credibilidad en muchos medios. "En un momento -ya va casi por una década- de crisis y transformación en los medios, no parece haber mejor modelo de negocio que la credibilidad. Frente a tanta posverdad, más y mejor fact-checking" (2017, p.16). 
Lotero Echeverri et al. (2018) perpetran un examen entre el fact-checking y las fake news, destacando esa competencia mediática en la búsqueda de la veracidad y que las páginas de chequeo se basan en un proceso que aspira a ser objetivo, transparente y replicable en todos sus pasos.

Frente a esto, es oportuno recordar lo que afirman autores como Pascual (2016) quien indica que, para obtener un buen producto es importante que todas las noticias sean verificadas, donde el comunicador esquive sus opiniones, sentimientos e intereses. Por lo cual se puede deducir que el fact-checking, nacido como mecanismo en la búsqueda de la veracidad, podría o no responder a procesos minuciosos como las prácticas, técnicas y el contexto en el que se aplique, lo que demanda un profundo estudio.

Pese a estas múltiples aristas, el fact-checking se potencializa en un mundo acelerado, con personas convertidas en prosumidores, viviendo una libertad de expresión, desafiando los límites de un ejercicio periodístico que se percibe muchas veces estancado. Es por ello que la Comisión Interamericana de Derechos Humanos está sumida en una búsqueda constante del monitoreo y regulaciones en el marco de la libertad de expresión en Internet (Marino, 2013), a partir de organizaciones que procuran controlar a los grandes intermediarios de la web.

De este modo, los portales de chequeo funcionan como entidades brindadoras de confianza frente a los filtros editoriales llevados dentro de las salas de redacción de los medios convencionales, las que han dejado cierta suspicacia en la profesión cayendo en sesgos, noticias falsas, en copyright como dice Smiers (2006) y prejuicios a la hora de informar. Rubén Darío Buitrón (2005) indica que estos problemas se dan porque "en las salas de redacción hace falta la pausa en medio del vértigo" (p. 69). Sin embargo, el fact-checking todavía es considerado como un concepto esquivo marcado por la presencia de usuarios indiferentes, cómplices en sus burbujas de opinión pública (Cervera, 2017) y de ese poder que ejercen las grandes esferas políticas (Castells, 2009).

En cuanto al tema de la opinión pública, si bien de acuerdo al contexto en el que se viva, los seres humanos están en derecho de la Libertad de Expresión, la Comisión Interamericana de Derechos Humanos, con el apoyo a la Relatoría Especial para la Libertad de Expresión, adopta la Declaración de Principios y en el literal 46 señala (Ávila, Ávila y Gómez, 2011): 
(...) este principio establece el estándar de la real malicia como ordenamiento legal a ser utilizado en la protección del honor de los funcionarios públicos o personas públicas. En la práctica dicho estándar se traduce en la imposición de sólo sanciones civiles en aquellos casos en que exista información falsa y producida con "real malicia"47, es decir producida con la intención expresa de causar un daño, o con pleno conocimiento de que dicha información era falsa, o con manifiesta negligencia en la búsqueda de la verdad o falsedad de las mismas. La carga de la prueba recae sobre quienes se sienten afectados por una información falsa o inexacta demostrando que el autor de la noticia procedió con malicia (p. 23).

El párrafo anterior deja claro que toda persona en particular, incluidos comunicadores, debe conocer los principios de Libertad de Expresión, concepto que en documentos se encuentra, pero que en la práctica ha acarreado muchos problemas, particularmente en el periodismo donde la búsqueda de dicha verdad se ve cada vez diluida y carente de credibilidad.

En síntesis, por un lado están los usuarios ávidos por crear y consumir información en una web casi anárquica. Paralelo a ello, se evidencia cómo el periodismo convencional va en su debacle dentro de las salsas de redacción y allí aparece el fact-checking que, pese a su intención de calificar el tipo de contenidos que circula entre la opinión pública, es cuestionado por su estructura en sí misma y por sus procesos metodológicos, donde se involucra el tipo de contrastación que utiliza para ponderar un tipo de contenido.

En esta lógica interesa poner en diálogo al portal Ecuador Chequea ${ }^{4}$. Su editora, Desirée Yépez, lo define como "un sitio no partidario que tiene como objetivo cotejar las declaraciones de políticos, líderes de la sociedad civil, personas públicas, medios de comunicación u otras instituciones formadoras de opinión" (Ecuador Chequea, s.f).

Este portal, que nació en octubre de 2016, expone que se amparan bajo dos organizaciones: Fundación Andina para la Observación Social y el

4 Primer medio dedicado a la verificación del discurso público y los contenidos engañosos que circulan en Internet. La plataforma es una iniciativa de Fundamedios. Ecuador Chequea detalla que es organización sin fines de lucro (http://www.ecuadorchequea.com/). 
Estudio de Medios (Fundamedios), este último nacido en contraposición de la Superintendencia de Comunicación (Supercom) organismo estatal que surgió para regular a los medios de comunicación y que 31 de julio, tras seis años de vida, fue cerrada.

Además de difundir su contenido en la página web www.ecuadorchequea. com, este portal divulga los chequeos por sus cuentas oficiales en Facebook, Instagram, Twitter y YouTube. También, mantiene una conexión más directa con su audiencia a través de WhatsApp y el correo info@ecuadorchequea.com.

Destacando esa competencia mediática en la búsqueda de la veracidad, Ecuador Chequea describe la metodología empleada en una página interior, disponible para cualquier usuario. Se basa en un proceso que aspira a ser objetivo, transparente y replicable en todos sus pasos.

En el portal se observa la forma de verificar contenidos. En sus inicios, y con el fin de hacerlo amigable a la audiencia, mostraba una imagen de un pollo, como especie de emoticón que al divulgar un contenido falso adquiría la forma de un pollo quemado. En la actualidad, la imagen es una pila o batería, en donde al catalogar como verdadera una información la pila es de color verde y está cargada, mientras que, si es falsa, sale descargada y con una señal roja.

Ecuador Chequea era, hasta septiembre del 2020, el único medio de verificación de información del país ahora surgió un portal denominado Ecuador Verifica que está conformado por medios de comunicación, organizaciones de la sociedad civil y universidades del país. Como este análisis se encuadra desde el jueves 22 de agosto de 2019 hasta el viernes 20 de septiembre de 2019, no se considerará al medio de comunicación actual, pero sí se plantea extender su investigación en otros aportes científicos.

En la página web, con sección metodología se especifican los niveles de veracidad cuyas categorías se describen en la Tabla 2. 
Tabla 2

Categorías de la metodología de Ecuador Chequea

\begin{tabular}{ll}
\hline Categorías & Conceptos \\
\hline Cierto & Las declaraciones de los sujetos políticos que son \\
& integralmente precisas, directamente congruentes con la \\
& fuente objetiva y no omiten datos ni limitan la contextualización \\
& de la temática en discusión. \\
& Dentro de nuestra iconografía, hemos sintetizado esta \\
& categoría con la figura de una batería completamente \\
& cargada. \\
Sí, pero & Los enunciados que incluyen información verificable o datos \\
& parcialmente veraces, por lo cual requieren mayores ejercicios \\
& de contextualización para determinar, con claridad y precisión, \\
& el nivel de acierto de la afirmación de un actor político. \\
Insostenible & Toda expresión que no permite identificar directamente \\
& los argumentos o las bases de datos de las cuales se \\
& haya desprendido dicho enunciado. En esta categoría no \\
& se adelanta criterio sobre la certeza o falsedad de una \\
& declaración hasta ubicar con precisión la fuente objetiva con la \\
& cual confrontar aquella información. \\
& Una batería a medio descargar es el símbolo en este nivel. \\
& Evocación de los apuros que pueden afrontar los sujetos \\
& políticos que hablan sin sustento. \\
& Una declaración que riñe abiertamente con el dato objetivo. \\
& Un argumento falaz. Sin más: una mentira comprobada... \\
& El uso de información falsa termina por agotar la batería. Por \\
& eso, la ciudadanía deberá estar «pilas» con la verdad. \\
Falso & Además, incluye la categoría FALSETA, con los cuales se \\
identifican los contenidos que se viralizan en Internet pero que \\
corresponden a desinformación. Imágenes alteradas, audios \\
con mensajes falsos, videos sacados de contexto, cadenas de \\
WhatsApp...
\end{tabular}

Nota. Elaboración propia basada en Ecuador Chequea (s.f). 
En Ecuador existen pocos estudios profundos relacionados con la metodología que aplica Ecuador Chequea y la periodicidad en sus publicaciones. Algunos trabajos investigativos han surgido de licenciaturas como el generado por Dols Hernández (2020) con un análisis de la metodología del fact-checking: Caso de Chequeado y Ecuador Chequea, o el de Saltos-Zambrano (2017) donde se destaca que no existen departamentos de chequeo dentro "de las salas de redacción que eviten la publicación-difusión de noticias erróneas o falsas" ( $p$. XXI). No obstante, se ha nombrado en varias investigaciones al portal Ecuador Chequea como un medio especializado dentro de la evolución del periodismo en el país (Vallejo \& Pérez, 2019) en la que incluso el portal mantiene una mirada direccionada a temas políticos y comicios electorales.

Otro estudio basado en Ecuador Chequea, relacionado al COVID-19 es el de Ramírez (2020), quien a modo de conclusión considera la importancia de combatir la desinformación:

Si el problema de la desinformación es tan evidente y los diagnósticos de la situación se hacen a todos los niveles, es momento de actuar y luchar contra la infodemia con la mejor arma: la educación y la difusión de herramientas para detectarlas (Ramírez, 2020, p.107).

Finalmente, un estudio que nace en España incorporó a Ecuador Chequea dentro de su análisis, donde evalúan las metodologías aplicadas por seis principales medios de verificación sudamericanos y españoles. Este importante aporte científico identifica que dichos portales presentan diferentes descripciones metodológicas, pero no varían en su totalidad y concepto, por lo que el autor las homologa en los cinco pasos siguientes:

1. Selección de la frase, afirmación o declaración pronunciada en un ámbito público o trino en redes sociales.

2. Evaluación de la relevancia, impacto social y repercusión en el debate público o viralización social.

3. Consulta a las fuentes: autor/a de la afirmación, fuentes oficiales, expertas, alternativas y recolección de bases de datos públicas y oficiales que permitan realizar la verificación. 
4. Contextualización de la afirmación realizando una labor de periodismo explicativo.

5. Otorgar una calificación con base en unas categorías establecidas (Rodríguez Pérez, 2020, p. 250).

Al ser Ecuador Chequea un medio relativamente nuevo, es pertinente observar su periodicidad de publicación y sus alcances de contrastación de información. Por este motivo, el presente trabajo tiene como objetivo exponer un estado de los estudios generados en relación al fact-checking y presentar un análisis de la periodicidad de contenidos que se publican en el portal web, indagando en las distintas fuentes a las que recurre el equipo de chequeo. Además, se propone detallar las temáticas que son chequeadas por el portal y se efectúa una comparación de la cantidad de fuentes que el medio emplea en unos ámbitos temáticos que en otros.

La investigación espera conocer: ¿Qué tan frecuentes son los contenidos que publica este portal? ¿Qué tipo de contenidos son los que más se chequean? ¿Cuáles son las fuentes a las qué más se recurre? Las respuestas a estas interrogantes ayudarían a dilucidar si realmente la cobertura de noticias revisadas por Ecuador Chequea va en correspondencia con la vertiginosa cantidad de noticias faltas y desinformación que conviven en las esferas públicas.

\section{MÉTODO}

Esta investigación tiene un enfoque cualitativo, es decir que "utiliza la recolección y análisis de los datos para afinar las preguntas de investigación o revelar nuevas interrogantes en el proceso de interpretación" (Hernández-Sampieri et al., 2014, p.7). El alcance es descriptivo no experimental y analiza dos aspectos: por un lado, la periodicidad de los contenidos que se publican en el portal web Ecuador Chequea y, por otro, el tipo de fuentes a las que recurre el equipo de chequeo de dicho portal.

Para obtener resultados, se aplicó la técnica de análisis observacional sistematizado de la plataforma web Ecuador Chequea, con una duración de 30 días, específicamente desde el jueves 22 de agosto de 2019 hasta el viernes 20 de septiembre de 2019. La revisión de la página es sincronizada, con un horario tope de observación hasta las 00 h00 de cada día. 
La tabla con la que se monitorea el portal es de elaboración propia. Sin embargo, además del orden numérico de publicación, fecha y titular de la noticia posteada en el portal, se toman en cuenta los tipos de fuentes según la metodología utilizada por Zommer (2015), precursora del fact-checking en Latinoamérica.

Aquellos tipos de fuentes que resalta Zommer (2015) son:

- $\quad$ Fuente original: quién dijo la frase.

- $\quad$ Fuente/s oficial/es: datos que genera el Estado.

- $\quad$ Fuentes alternativas: datos que producen otros organismos, organizaciones o instituciones.

Además, se analizan las temáticas que son chequeadas por el portal y se compara si el medio emplea más fuentes en unos ámbitos temáticos que en otros.

\section{RESULTADOS}

Durante los 30 días de análisis de publicaciones en el portal web Ecuador Chequea, se puede evidenciar que la generación de contenidos no es secuencial ni permanente, y que el equipo de dicho medio realizó 25 publicaciones, como se evidencia en la Tabla 3, es decir, un promedio de 1.2 notas por día. Hubo días en los que no publicaron nada, pero en otros se difundieron entre dos y tres notas en el lapso de las 24 horas. Dichas notas eran luego posteadas en la cuenta de Facebook, que tiene, hasta el 22 de septiembre de 2019, a 10861 seguidores en un país con 17.620.846 millones de habitantes (INEC, 2020). Esto devela que Ecuador Chequea es un medio que probablemente se ve abrumado con la cantidad de información que gira en torno a la opinión pública lo cual, si bien sus intentos son un aporte hacia la veracidad de los hechos, no terminan de cubrir la necesidad de determinar la calidad de información que está consumiendo la audiencia. Aun así, este portal de chequeo no se somete a la inmediatez que gobierna el mundo mediático, sino que se frena para meditar e indagar el surgimiento de una información, lo cual requiere tiempo y dedicación. 
Tabla 3

Análisis del portal web Ecuador Chequea, clasificación según temáticas chequeadas

\begin{tabular}{lcc}
\hline Contenidos & Cantidad & Porcentaje \\
\hline Educación & 2 & $8 \%$ \\
Seguridad & 1 & $4 \%$ \\
Ecología & 5 & $20 \%$ \\
Migración & 2 & $8 \%$ \\
Política & 13 & $52 \%$ \\
Deportes & 2 & $8 \%$ \\
\hline TOTAL & 25 & $100 \%$ \\
\hline
\end{tabular}

Sin embargo, ese tiempo y dedicación deben verse reflejados en la calidad de contrastación que Ecuador Chequea emplea para presentar una información verificada. De acuerdo al tipo de fuentes que utilizan en la metodología expuesta por Zommer (2015), en esta muestra de respaldo de fuentes durante los 30 días de análisis se puede constatar en la Tabla 4 que solo recurrieron a 8 fuentes originales, mientras que el mayor número fueron con fuentes oficiales, llegando a consultar a $39 \mathrm{y}$, en las fuentes alternativas, se recurrió a 24 .

Tabla 4

Análisis del portal web Ecuador Chequea, clasificación según la fuente

\begin{tabular}{lcc}
\hline Fuentes & Total & Porcentaje \\
\hline Número de Fuente & 8 & $11.27 \%$ \\
Original & 39 & $54.93 \%$ \\
Fuentes Oficiales & 24 & $33.8 \%$ \\
Fuentes Alternativas & 71 & $100 \%$ \\
\hline TOTAL
\end{tabular}

Consultar a la fuente original es uno de los pasos obligatorios en la metodología del IFCN, sin embargo, de las 25 noticias, solo en 8 de ellas se consultó al autor o la autora de la (des)información. Lo anterior acarrea dudas en el proceso de chequeo del portal; a pesar de ello, dentro de las noticias chequeadas existen contenidos que no precisamente debían ser confirmados o no por la fuente generadora de la información o de quién se hablaba. Por ejemplo: "Negocio de winchas NO es del sobrino de Jorge 
Yunda \#FALSETA"; en esta información, publicada el 27 de agosto del 2019, se puede evidenciar que Ecuador Chequea no consultó directamente al sobrino de Jorge Yunda, sino que acudió a una fuente oficial como la Agencia Metropolitana de Tránsito para corroborar si era cierto o no. Por casos similares a estos es que no es mayor el índice de consulta a la fuente original.

Por otro lado, en otros casos particulares como: "\#FALSETA: Ministerio del Interior autoriza emisión de permisos para portar armas", debido a que se trataba de una tergiversación de la información dada por la autoridad, Ecuador Chequea recurre a consultar a María Paula Romo, en ese entonces Ministra de Gobierno.

En los 30 días de análisis se puede comprobar que el medio recurrió a un total de 71 fuentes. En futuros estudios, habría que estimar el hecho de conocer el tipo de rutinas productivas generadas, con el fin de revelar ciertas aristas, como si el equipo de chequeo es más de escritorio o se hace un tipo de cobertura para constatar, contrastar y conversar personalmente con las fuentes. Esto aunque en la Tabla 5 se puede observar que existe un mayor chequeo por noticias nacionales (18), que por las internacionales (7).

Tabla 5

Análisis del portal web Ecuador Chequea, clasificación según clasificación nacional e internacional

\begin{tabular}{lcc}
\hline \multicolumn{1}{c}{ Noticias } & Número & Porcentaje \\
\hline Internacionales & 7 & $28 \%$ \\
Nacionales & 18 & $72 \%$ \\
\hline TOTAL & 25 & $100 \%$ \\
\hline
\end{tabular}

Palau-Sampio (2018) expresa que las fuentes son un factor crucial en estos portales, porque el veredicto final y su credibilidad dependen en gran medida de la variedad y confiabilidad de las fuentes. El autor analizó algunos portales latinoamericanos, para conocer cómo se procesan y evalúan las afirmaciones públicas que se someten a evaluación.

En comparación con los hallazgos del trabajo Palau-Sampio (2018) se puede evidenciar que existe una coincidencia con el presente estudio en cuanto a la cantidad de fuentes que suelen emplearse por cada información 
analizada. Hay casos donde el portal Ecuador Chequea utiliza entre tres o más fuentes de información para verificar el contenido, mientras que existen otras notas que se basan en dos o menos fuentes, y otro grupo menor que solo tiene una o ninguna fuente; en consecuencia, este estudio concuerda en que es difícil definir el número óptimo de fuentes requeridas, no obstante, el uso de una sola opción es muy limitado, sobre todo si se trata de portales de chequeo.

Haciendo una ponderación entre la cantidad de fuentes a las que Ecuador Chequea recurrió en relación con las 25 notas chequeadas, existe un promedio de 2.84 fuentes consultadas por notas.

Por otra parte, los temas mayormente verificados fueron de Política, seguido por las categorías de Ecología y Migración (ver Tabla 6). Aun así, en la página web se puede ver que las temáticas se ven marcadas por la viralidad de informaciones dudosas y que rondan la esfera pública, tal como sucede en portales de chequeo como Maldita y Newtral, evaluados por otros investigadores. Por lo tanto, la periodicidad no es sistematizada y, como se explicó, en esta muestra se evidencia que hubo días en los que no se difundió ningún contenido, mientras que en otros se difundieron hasta tres publicaciones. Aunque no se evidencia un patrón de publicación en particular, se pude destacar que Ecuador Chequea pone mayor atención a casos relacionados a la política.

Tabla 6

Análisis del portal web Ecuador Chequea, clasificación según cantidad de fuentes de acuerdo a cada ámbito temático

\begin{tabular}{lcc}
\hline Contenidos & $\begin{array}{l}\text { Cantidad de fuentes de acuerdo a } \\
\text { cada ámbito temático }\end{array}$ & Porcentaje \\
\hline Educación & 3 & $4.23 \%$ \\
Seguridad & 2 & $2.82 \%$ \\
Ecología & 9 & $12.68 \%$ \\
Migración & 9 & $12.68 \%$ \\
Política & 42 & $59.15 \%$ \\
Deportes & 6 & $8.45 \%$ \\
\hline TOTAL & 71 & $100 \%$ \\
\hline
\end{tabular}




\section{DISCUSIÓN}

Sobre esa avalancha de información que existe en Internet con miles y millones de usuarios devenidos en consumidores, se evidencia una lucha cuasi utópica hacia la búsqueda de detectar el grado de validez de la información publicada, como en el caso de Ecuador Chequea. Tener poca periodicidad en la publicación de contenidos que faciliten la corroboración de informaciones termina siendo una debilidad, como se demuestra en los resultados, ante la cantidad de información que es publicada a diario tanto en redes, como en medios convencionales.

El uso de diferentes fuentes periodísticas en ciertas publicaciones y el escaso número en otras, evidencia cierta falta de credibilidad, aquello no es precisamente un tema explícito del portal de Ecuador Chequea, pues estudios como García-Galera et al. (2020) y Palau-Sampio (2018) reflejan que los procedimientos metodológicos donde se incluye la consulta a la fuente podrían ser replanteados con mejores bases epistemológicas que brinden al periodismo de verificación una mayor consolidación.

Es evidente que la inmediatez en medios periodísticos convencionales prevalece, adulterando la credibilidad de sus contenidos. Por eso, está distante, al menos en Latinoamérica, que se apliquen metodologías donde el chequeo sea parte de los filtros editoriales en las redacciones de los medios previo a que un contenido sea publicado, tal como lo realizan las revistas Piaui de Brasil y The New Yorker.

Se puede inferir que los usuarios que visitan la página podrían ser personas interesadas en eso, en saber qué hecho es real, pero no son todos los ecuatorianos, ya que los usuarios en muchas ocasiones "ven lo que quieren ver", "consumen lo que desean consumir" y "creen en lo que desean creer", volviendo nuevamente a aquella modernidad líquida a la que se refiere Bauman (2015). Quizás estos son mecanismos que generan cierta seguridad en quienes consumen o siguen este tipo de medios, pero no está aquí la solución a un problema latente y de mucha magnitud, mucho menos si el proceso de chequeo es artesanal, es decir, no hay un apoyo tecnológico en gran medida, por ejemplo, un tipo de inteligencia artificial que evite los sesgos en el proceso de calificación de una noticia verdadera, falsa, insostenible, etc. 
Por otro lado, interesa destacar a aquellos autores que sí creen en este proceso de selección de noticias, como Zommer (2015), quien bajo un orden y sistematización explica los procesos de chequeo en su inminente búsqueda por la veracidad de los hechos y de, quizás, salvar a la sociedad de las fake news. Lo mismo explican Lotero Echeverri et al. (2018) quienes ponen de ejemplo fact-checking como un "guerrero" ante las fake news, destacando esa contención mediática en la búsqueda de la veracidad.

Por todo lo expuesto, y tras analizar y contrastar diversas teorías enmarcadas en el tema citado, podría señalarse que en un mundo mediatizado es importante que las funciones del fact-checking se vean fuertemente apoyadas por procesos de Inteligencia Artificial, en los que desde sus logaritmos sean mínimos los márgenes de error para corroborar hechos. Ecuador Chequea no es aquí la excepción.

Este tipo de acciones podrían dar cabida a un verdadero posicionamiento del fact-checking en su imperiosa investigación por aclarar sucesos. Está claro que detrás de este proceso no estarían solo periodistas, sino gente especializada en análisis de sistemas y distintas disciplinas que comiencen a equiparar el ritmo acelerado en la publicación de noticias de los medios de comunicación, con el lento y en ocasiones sesgado, proceso de las entidades de fact-checking.

Este trabajo investigativo es un aporte a la ciencia, con miras a futuras pesquisas en las que se puedan plasmar nuevas olas de análisis relacionados a la comparación de la dinámica de trabajo de las plataformas de chequeo e incluso, hacia la comparación de las rutinas productivas llevadas a cabo entre medios convencionales y los de fact-checking.

\section{REFERENCIAS}

Ávila, M. P., Ávila, R., \& Gómez, G. (2011). Libertad de expresión: debates, alcances y nueva agenda. V\&M Gráficas.

Bauman, Z. (2015). Modernidad líquida. Fondo de cultura económica.

Becerra, M., \& Marino, S. (2014). Chequeando a Chequeado La Voz Pública. [online] Chequeado.com. https://chequeado.com/wp-content/ uploads/2015/02/CHEQUEANDO-a-CHEQUEADO-1.pdf 
Buitrón, R. D. (2005). Seis reflexiones sobre periodismo impreso y periodismo digital. Sala de Prensa, 79.

Busaniche, B. (2005). Las ideas y las cosas: la riqueza de las ideas y los peligros de su monopolización. Un mundo patentado, 68-82.

Castells, M. (2009). Comunicación en la era digital. Comunicación y Poder. Alianza Editorial.

Cervera, J. (2017). El futuro del periodismo es cíborg. Cuadernos de periodistas: revista de la Asociación de la Prensa de Madrid. (34), 102-109.

Constine, J. (2012). ¿Qué tan grande son los datos de Facebook? 2.5 mil millones de piezas de contenido y más de 500 terabytes ingeridos todos los días. Tech Crunch. https://techcrunch.com/2012/08/22/ how-big-is-facebooks-data-2-5-billion-pieces-of-content-and-500-terabytes-ingested-every-day/

Dols Hernández, A. (2020). Análisis de la metodología del fact-checking: Caso de Chequeado y Ecuador Chequea (tesis de licenciatura). Recuperado de Universidad Miguel Hernández http://193.147.134.18/ bitstream/11000/6619/1/PER_TFG_DOLS_HERNANDEZ_ALBA.pdf

Echevarría, B. (2017). Más 'fact-checking' contra la posverdad. Cuadernos de periodistas, 33, 9-16.

Ecuador Chequea. (s.f). Quiénes somos. http://www.ecuadorchequea. com/quienes-somos/

Elisabeth, J. (2014). Who are you calling a fact checker? American Press Institute. https://www.americanpressinstitute.org/fact-checking-project/ fact-checker-definition/

García-Galera, M. D. C., Del Hoyo-Hurtado, M., \& Blanco-Alfonso, I. (2020). Desinformación e intención comunicativa: Una propuesta de clasificación de fake news producidas en entornos periodísticos profesionales. Revista Mediterránea De Comunicación: Mediterranean Journal of Communication, 11(2), 105-118. https://www.doi.org/10.14198/ MEDCOM2020.11.2.16 
Graves, L. (2018). Boundaries not drawn. Journalism Studies, 19(5), 613631. https://doi.org/10.1080/1461670x.2016.1196602

Hernández-Sampieri, R., Fernández-Collado, C., \& Baptista-Lucio, P. (2014). Metodología de la Investigación, Sexta Edición México. DF, Editores, SA de CV.

IFCN Code of Principles. (2020). Verified signatories of the IFCN code of principles. https://www.ifcncodeofprinciples.poynter.org/signatories

INEC. (2020). Retrieved 10 December 2020, from https://www.ecuadorencifras.gob.ec/estadisticas/

Unión Internacional de las Telecomunicaciones. (ITU). (2018). Informe sobre Medición de la Sociedad de la Información, Resumen analítico 2018. https://www.itu.int/en/ITU-D/Statistics/Documents/publications/misr2018/MISR2018-ES-PDF-S.pdf

Lotero-Echeverri, G., Romero-Rodríguez, L. M., \& Pérez-Rodríguez, M. A. (2018). Fact-checking vs. Fake news: Periodismo de confirmación como recurso de la competencia mediática contra la desinformación. Index.comunicación: Revista científica en el ámbito de la Comunicación Aplicada, 8(2), 296-316.

Luna, G. (2015). Inmediatez Informativa, el desafío del periodista digital. En Irigaray, F. (Ed.) Reflexiones móviles: el periodismo en la era de la movilidad (pp. 33-50). UNR Editora.

Marino, C. B. (2013). Libertad de expresión e Internet. Oficina de la Relatoría Especial para la Libertad de Expresión de la Comisión Interamericana de Derechos Humanos. Organización de los Estados Americanos..

Martini, S. (2000). Acontecimiento y noticia, Las fuentes periodísticas, El estudio de la noticiabilidad. En S. Martini Periodismo, noticia y noticiabilidad (pp. 29-44, 45-72, 73-102) . Grupo Editorial Norma.

Fundación Gabo. (2020). Más de 100 sitios para verificar desinformación sobre el covid-19. https://fundaciongabo.org/es/noticias/ mas-de-100-sitios-para-verificar-desinformacion-sobre-el-covid-19 
Palau-Sampio, D. (2018). Fact-checking y vigilancia del poder: La verificación del discurso público en los nuevos medios de América Latina. Comunicación y Sociedad, 31(3), 347-365. https://www.doi. org/10.15581/003.31.3.347-365

Pascual, M. (2016). Política y propaganda: medios de comunicación y opinión pública. Editorial UOC.

Pons, P. (31 de julio de 2018). 2.500 millones personas usan, al menos, una 'app' de la familia Facebook. La Vanguardia. https://www.lavanguardia.com/tecnologia/20180731/451170165167/facebook-usuarios-redes-sociales-tecnologia-portada.html

Ramírez, K. B. (2020). Visualización del contexto de las fake news para entender la infodemia. \#PerDebate, 4(1). https://doi.org/10.18272/ pd.v4i1.1995

Rodríguez-Pérez, C. (2020). Una reflexión sobre la epistemología del fact-checking journalism: Retos y dilemas. Revista De Comunicación, 19(1), 243-258.

Salaverría, R., Buslón, N., López-Pan, F., León, B., López-Goñi, I., \& Erviti, M. (2020). Desinformación en tiempos de pandemia: Tipología de los bulos sobre la Covid-19. El Profesional De La Información (EPI), 29(3), e290315. https://doi.org/10.3145/epi.2020.may.15

Saltos-Zambrano, M. N. (2017). La importancia y necesidad de un departamento de fact checking (verificación) en los medios de comunicación. Estudio desde la experiencia del portal Ecuador Chequea. Repositorio Digital UCSG http://repositorio.ucsg.edu.ec/handle/3317/8959

Smiers, J. (2006). Un mundo sin copyright: artes y medios en la globalización. Medialab Prado. https://www.medialab-prado.es/actividades/ un-mundo-sin-copyright-artes-y-medios-en-la-globalizacion

Stencel, M. (11 de junio de 2019). Number of fact-checking outlets surges to 188 in more than 60 countries. Poynter. https://www. poynter.org/ fact-checking/2019/number-of-fact-checking-outlets-surges-to-188in-more-than-60-countries/ 
Ufarte-Ruiz, M., Peralta-García, L., \& Murcia-Verdú, F. (2018). Fact checking: Un nuevo desafío del periodismo. El Profesional De La Información, 27(4), 733-741. https://www.doi.org/10.3145/epi.2018.jul.02

Uscinski, J. E. (2015). La epistemología de la verificación de hechos (todavía es ingenua): Dúplica a Amazeen. Revisión crítica, 27 (2), 243-252.

Uscinski, J. E., \& Butler, RW. (2013). La epistemología de la verificación de hechos. Revisión crítica, 25 (2), 162-180.

Vallejo, R. M., \& Pérez, B. Z. (2019). Historia, evolución y desafíos del periodismo digital en el Ecuador. Textos y Contextos (segunda época), (18), 11-24.

Vizoso, Á., \& Vázquez-Herrero, J. (2019). Plataformas de fact-checkingenespañol. características, organización y método. Comunicación y Sociedad, 32(1), 127-144. https://www. doi.org/10.15581/003.32.1.127-144

Wardle, C. (2017). Fake news. It's complicated. First Draft. http://bit. ly/39jjmc4

Wolf, M. (1997). Los emisores de noticias en la investigación sobre comunicación. Zer, 3. Revista de comunicaciones. Universidad del País Vasco.

Zommer, L. (2014). El boom del fact checking en América Latina. Aprendizajes y desafíos del caso de Chequeado. Chequeando.

Zommer, L. (2015). El boom del fact checking y la vuelta a las fuentes. La Crujía Ediciones. 
